\title{
Emergência de Physalis Angulata L. em resposta à temperaturas e períodos de imersão em água
}

Physalis angulata L. é um arbusto anual que pertence à família Solanaceae com grande ação medicinal. Essa cultura da flora Amazônica é fonte de vitaminas A e C além de ser rica também em fósforo, ferro, carotenoides e flavonoides. O trabalho teve como objetivo foi avaliar a emergência de plântulas de $P$. angulata em função da temperatura e do período de imersão em água. O experimento foi conduzido em esquema fatorial $3 \times 2$, sendo: temperaturas de imersão em água de $40^{\circ} \mathrm{C}, 60^{\circ} \mathrm{C}$ e $80^{\circ} \mathrm{C}$ e períodos de imersão de 5 e 10 minutos. $\mathrm{O}$ tratamento controle foi a imersão das sementes em água, em temperatura ambiente, durante 24 h. As variáveis de resposta foram: emergência percentual, índice de velocidade de emergência, tempo médio de emergência e velocidade média de emergência. Os resultados foram submetidos à análise de variância e comparados pelo teste de Tukey $(p<0,05)$. Todas as variáveis foram influenciadas pelos tratamentos, com melhor resposta para o controle, com percentual de emergência igual a $74 \%$, e pior para a imersão em água a 80 으 durante 10 minutos que apresentou $0,4 \%$ de emergência. A imersão das sementes de $\mathrm{P}$. angulata em água, sob temperatura ambiente durante $24 \mathrm{~h}$ é recomendável na emergência de plântulas, sendo também destacadas a imersão em água a $40 \circ \mathrm{C}$ por 10 minutos e a 60 으 por cinco minutos.

\section{Physalis Angulata L. emergency in response to temperatures and water immersion periods}

\begin{abstract}
Physalis angulata L. is an annual shrub that belongs to the Solanaceae family with great medicinal action. The aim was to evaluate the emergence of P. angulata seedlings as a function of temperature and the period of immersion in water. The experiment was conducted in a $3 \times 2$ factorial scheme, being: immersion temperatures in water of $40^{\circ} \mathrm{C}, 60^{\circ} \mathrm{C}$ and $80^{\circ} \mathrm{C}$ and periods of immersion of 5 and 10 minutes. The control treatment was the immersion of the seeds in water at room temperature for $24 \mathrm{~h}$. The response variables were percentage emergence, emergence speed index, mean time of emergence and average speed of emergence. The results were submitted to analysis of variance and compared by the Tukey test $(p<0.05)$. All variables were influenced by treatments, with a better response to the control, with an emergence percentage equal to $74 \%$, and worse for immersion in water at $80 \circ \mathrm{C}$ during 10 minutes, which presented $0.4 \%$ emergence. The immersion of the seeds of P. angulata in water, at room temperature for $24 \mathrm{~h}$ is recommended in the emergence of seedlings, being also emphasized the immersion in water at $40 \circ \mathrm{C}$ for 10 minutes and at $60 \circ \mathrm{C}$ for five minutes.
\end{abstract}

Keywords: Seed Analysis; Camapu; Solanaceae.

Wendel Valter da Silveira Pereira (D) Universidade Federal Rural da Amazônia, Brasil http://lattes.cnpq.br/9704897001117996 http://orcid.org/0000-0002-7958-6758 wendelvalter@gmail.com

Deivison Rodrigues da Silva (D)

Universidade Federal Rural da Amazônia, Brasil http://lattes.cnpq.br/8106102331831281 http://orcid.org/0000-0001-5607-084X deivisonrodrigues01@live.com

Jessivaldo Rodrigues Galvão (iD Universidade Federal Rural da Amazônia, Brasil http://lattes.cnpq.br/0013591065769741 http://orcid.org/0000-0003-4242-6555 jessigalvao50@gmail.com
Flavio Henrique Santos Rodrigues (iD) Universidade Federal Rural da Amazônia, Brasil http://lattes.cnpq.br/9173056304877884 http://orcid.org/0000-0002-0080-5453 flaviohsrodrigues2@gmail.com

Mauro Junior Borges Pacheco (D)

Universidade Federal Rural da Amazônia, Brasil http://lattes.cnpq.br/0756046215703468 http://orcid.org/0000-0001-6024-7054 mauro.jr720@gmail.com

Wallasi Washington Silva da Silva

Universidade Federal Rural da Amazônia, Brasil http://lattes.cnpq.br/4227406303938287 wallasiwashington@gmail.com
Gabriela Vilhena de Almeida (iD

Universidade Federal Rural da Amazônia, Brasil http://lattes.cnpq.br/1923584857795627 http://orcid.org/0000-0001-5712-8062 gabrielaalmeida16@hotmail.com

Arley Thoncy Almeida Cardoso (iD) Universidade Federal Rural da Amazônia, Brasil http://lattes.cnpq.br/8200523931549713 http://orcid.org/0000-0003-4976-0249 arley.thoncy@gmail.com

\section{Referencing this:}

PEREIRA, W. V. S.; SIVA, D. R.; GALVÃO, J. R.; RODRIGUES, F. H. S.; PACHECO, M. J. B.; SILVA, W. W. S.; ALMEIDA, G. V.; CARDOSO, A. T. A.. Emergência de Physalis Angulata L. em resposta à temperaturas e períodos de imersão em água. Revista Ibero Americana de Ciências Ambientais, v.11, n.1, p.333-340, 2020. DOI: http://doi.org/10.6008/CBPC2179-6858.2020.001.0030 


\section{INTRODUÇÃO}

O arbusto anual Physalis angulata L., conhecido na região norte do Brasil como camapu, pertence à família Solanaceae e é amplamente distribuído nas regiões tropicais e subtropicais no mundo (KUSUMANINGTYAS et al., 2015). É uma espécie que vem sendo alvo de muitos estudos, motivados pela riqueza dos frutos em nutrientes, proteínas, aminoácidos essenciais, vitaminas, carotenoides e fenóis (PATEL et al., 2011). O processo germinativo se dá com a quebra do tegumento pela radícula sendo uma das etapas mais importantes para o ciclo de vida das plantas. Os tratamentos de pré-germinação envolvendo o vigor, a eficácia de germinação e condicionamento de sementes são bastante aplicados para melhorar a qualidade de sementes e o estabelecimento das plantas cultivadas (SOUZA et al., 2013).

A Temperatura merece grande atenção, em razão da sua influência na velocidade de absorção de água, assim como apresenta papel fundamental nas reações bioquímicas que ocorrem no interior da semente e ser determinantes no processo germinativo (CARVALHO et al., 2012). É uma das variáveis ambientais que mais influência na germinação de sementes (KOGER et al., 2004) e tende a diferir entre as culturas em relação ao melhor potencial germinativo. O conhecimento sobre a resposta das sementes à temperatura é primordial, pois torna possível compreender a amplitude de tolerância das espécies em relação à temperatura, bem como as condições climáticas nas quais as culturas podem germinar e se estabelecer de maneira adequada (MOTSA et al., 2015).

A germinação de sementes é diretamente relacionada a fatores como disponibilidade de água e temperatura. Para espécies não dormentes, como é o caso de $P$. angulata, a água geralmente é um fator limitante, que influencia aspectos como velocidade e uniformidade de germinação e emergência. 0 abastecimento apropriado de água nas sementes é fundamental para processos como mobilização de reservas, liberação de energia, e atividade enzimática e hormonal em plantas (BASKIN et al., 2014).

A imersão de sementes em água quente é um método que demanda baixo custo, pode ser utilizado em larga escala (CASTRO et al., 2017), proporciona menores riscos de toxidade e é mais prático em relação a métodos químicos e mecânicos de tratamento, especialmente para sementes pequenas (BENEDITO et al., 2017), como é o caso de $P$. angulata. Levando em consideração a importância medicinal atribuída à essa espécie, além da escassez de estudos sobre seus aspectos de cultivo e germinação, o objetivo foi avaliar a emergência de $P$. angulata em função da temperatura e tempo de imersão em água.

\section{MATERIAIS E MÉTODOS}

No Laboratório de análises de solo da Universidade Federal Rural da Amazônia (UFRA), foram separadas 100 sementes de Physalis angulata L., coletados de frutos, em estágio final de maturação, caídos sobre o solo, em área de vegetação espontânea. As mesmas foram acondicionadas em sacos de tecido perfurados e, imersos em água destilada mantida sob aquecimento em banho-maria regulado às temperaturas de 40,60 e $80^{\circ} \mathrm{C}$, onde permaneceram expostas por 5 e 10 minutos. Após cada período de exposição, os sacos contendo as sementes foram retirados da água e abertos, sendo as sementes colocadas 
para secar naturalmente sobre papel toalha em condições de laboratório $\left(25 \pm 2^{\circ} \mathrm{C}\right)$.

Os tratamentos, compostos pelas temperaturas e tempo de exposição, foram alocados em delineamento experimental inteiramente casualizado, distribuídos em esquema fatorial 3x2, em 5 duplicatas cada um. Adotou-se um tratamento controle, caracterizado pela imersão das sementes em água natural durante 24 h. $O$ ensaio foi conduzido, utilizando-se 200 sementes, subdivididas nas cinco repetições.

Os tratamentos foram assim distribuídos: Controle (imersão em água natural por 24 horas); imersão em água a 40 으, durante cinco minutos (T40P5); imersão em água a 40ํㅡ, durante dez minutos (T40P10); imersão em água a 60드. durante cinco minutos (T60P5); imersão em água a 60드. durante dez minutos (T60P10); imersão em água a 80ํㅡ, durante cinco minutos (T80P5); e imersão em água a 80ㄷ, durante dez minutos (T80P10).

Os parâmetros avaliados foram: percentual de emergência, índice de velocidade de emergência, tempo médio de emergência e velocidade média de emergência. O percentual de emergência foi calculado através da equação proposta nas regras para análise de sementes (BRASIL, 2009). O índice de velocidade de emergência realizado de acordo com a fórmula proposta por Maguire (1962). O tempo médio de emergência a partir da fórmula proposta por Edmond et al. (1958) e a velocidade média de emergência foi calculada através da fórmula proposta por Kotowiski (1926). Os resultados foram submetidos à análise de variância, sendo as médias comparadas pelo teste de Tukey se selecionando o modelo significativo de $5 \%$. As análises foram realizadas no software computacional SISVAR (FERREIRA, 2011).

\section{RESULTADOS}

Com os resultados do percentual de emergência (Figura 1), verificou-se que ocorreu efeitos moderados dos tratamentos térmicos e tempo de exposição das sementes, sendo os mesmos inferiores ao tratamento com exposição a água natural por 24 horas, com percentual de $74 \%$. Verifica-se que o percentual reduz drasticamente quando as sementes são expostas a elevação da temperatura da água.

As imersões a $40 \circ \mathrm{C}$ por dez minutos ( $>50 \%$ de emergência) e a $60 \circ \mathrm{C}$ durante cinco minutos (aproximadamente 50\% de emergência) promoveram resultados semelhantes, porém bastante significativos com relação ao tempo de imersão. Efeitos bem reduzidos na germinação foram observados com o maior tempo de exposição à temperatura de $600^{\circ}$ C. Efeitos da interação entre a temperatura e tempo de imersão, constatou-se para o índice de velocidade de emergência. O tratamento controle proporcionou o melhor índice, com 4,51 (Figura 2).

Verificou-se interação significativa entre as temperaturas e os períodos de imersão com relação ao tempo médio de emergência, sendo observados maiores resultados para a imersão em água a 80 ํㅡ. As imersões em água a 40 e $60{ }^{\circ} \mathrm{C}$ durante 5 e 10 minutos não diferiram estatisticamente. 0 tratamento controle apresentou o menor tempo médio de emergência (Figura 3). 


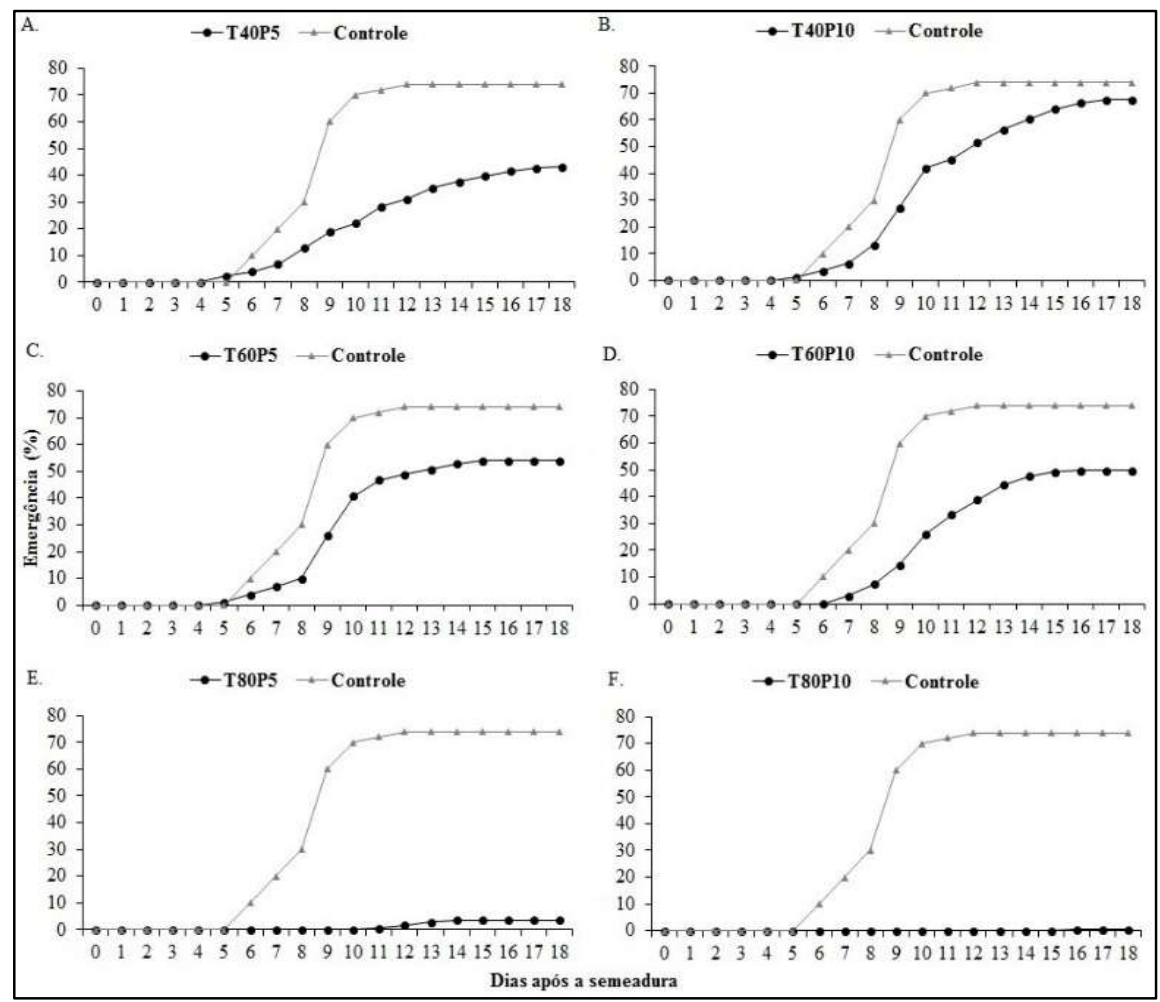

Figura 1: Emergência percentual de plântulas de Physalis angulata L. em função da imersão em água a 40ํ durante 5 minutos - T40P5 (A), 40C durante 10 minutos - T40P10 (B), 60C durante 5 minutos - T60P5 (C), 60C durante 10 minutos - T60P10 (D), 80ㄷ durante 5 minutos - T80P5 (E), 80ㄷ durante 10 minutos - T80P10 (F) e comparação ao tratamento controle.

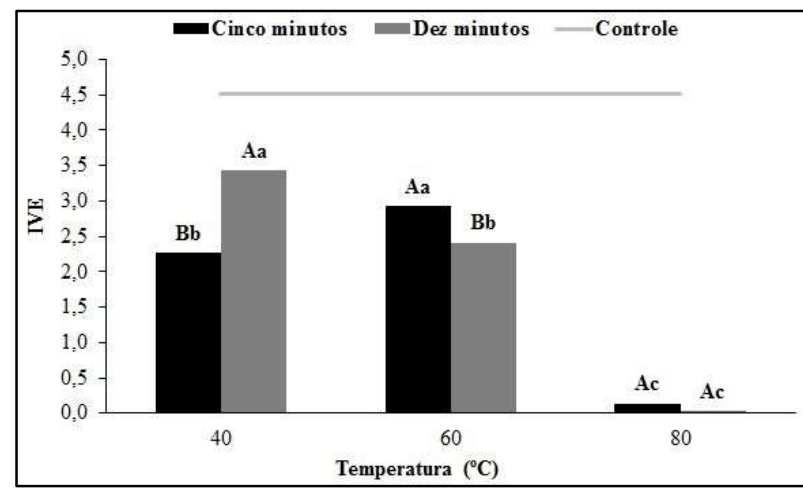

Figura 2: Índice de velocidade de emergência (IVE) de Physalis angulata L. em função da temperatura e do período de imersão em água. Letras maiúsculas comparam períodos e minúsculas comparam temperaturas de imersão pelo teste de Tukey $(p<0,05)$.

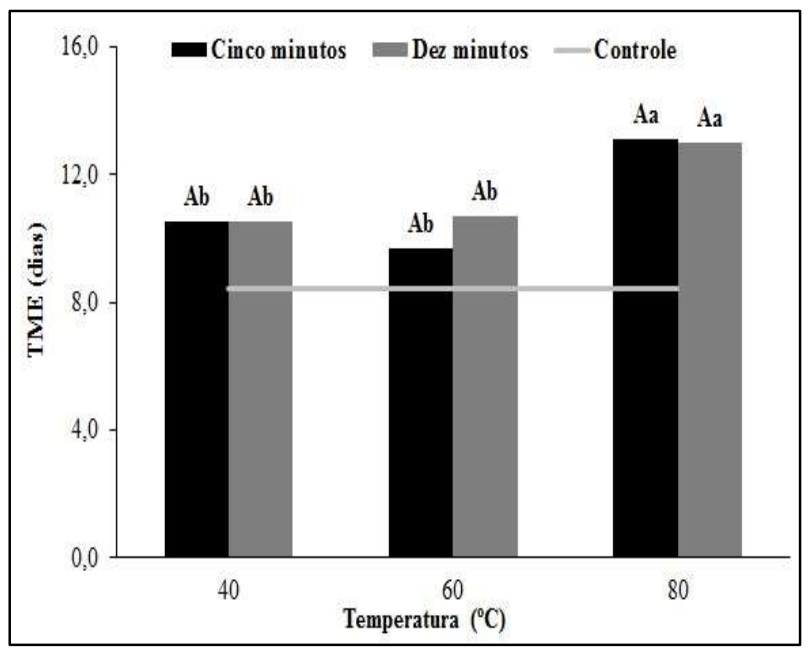

Figura 3: Tempo médio de emergência (TME) de Physalis angulata L. em função da temperatura e do período de imersão em água. Letras maiúsculas comparam períodos e minúsculas comparam temperaturas de imersão pelo teste de Tukey $(p<0,05)$.

A velocidade média de emergência sofreu influência da interação entre as temperaturas e os períodos de imersão em água. Os períodos de 5 e 10 minutos não diferiram entre si para as temperaturas de 40 e $60^{\circ} \mathrm{C}$. Os períodos de imersão sofreram efeitos significativos com a temperatura de $80^{\circ} \mathrm{C}$ (Figura 4 ). 


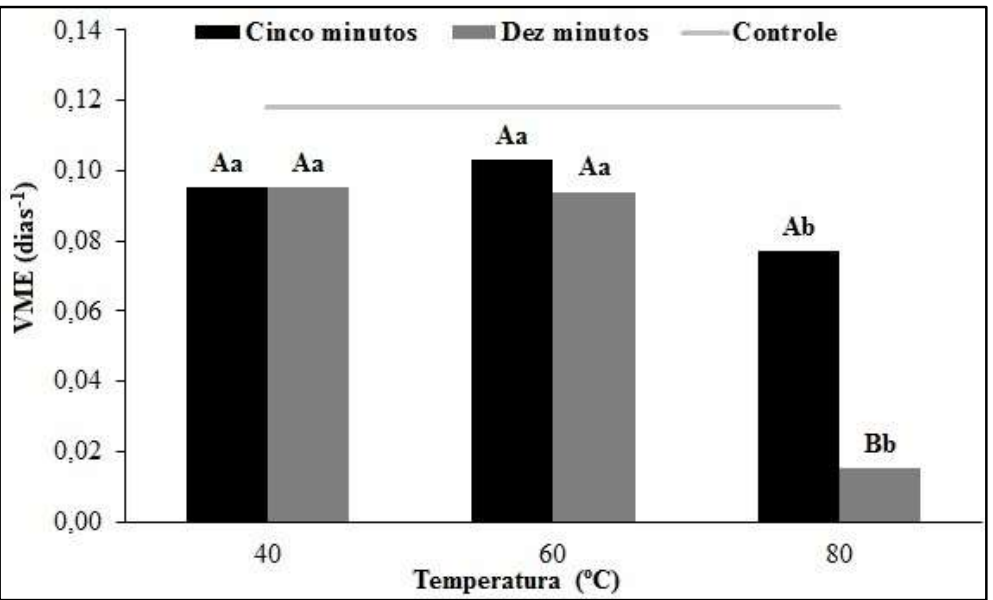

Figura 4: Velocidade média de emergência (VME) de Physalis angulata L. em função da temperatura e do período de imersão em água. Letras maiúsculas comparam períodos e minúsculas comparam temperaturas de imersão pelo teste de Tukey $(p<0,05)$.

\section{DISCUSSÃO}

A imersão das sementes em água sob temperatura ambiente por $24 \mathrm{~h}$ proporcionou o maior percentual de sementes emergidas, o que pode estar relacionado ao maior tempo de exposição das sementes em água à temperatura ambiente. $\mathrm{O}$ abastecimento de água nas sementes reidrata os tecidos, estimulando as atividades metabólicas, promovendo fornecimento energético e nutricional e favorecendo o desenvolvimento embrionário (CARVALHO et al., 2012), elevando o percentual germinativo.

A maior temperatura reduziu significativamente a emergência percentual, independente do período de imersão em água. Esses resultados podem estar relacionados aos efeitos negativos da alta temperatura nos mecanismos fisiológicos e no tecido embrionário das sementes, provocando a redução no percentual de emergência de plântulas (CARNEIRO et al., 2010).

A redução do percentual de emergência com o aumento da temperatura, foi constatada em outros estudos. Alguns métodos foram estudados na superação de dormência de sementes de Bauhinia divaricata L. ocorrendo diminuição significativa no percentual germinativo para a imersão em água a 80ำ, comparada às demais imersões de 50,60 e 70C (ALVES et al., 2004). Da mesma forma, a emergência de plântulas de Capsicum baccatum L. apresentou percentual de emergência significativamente menor em função da imersão em água a $70^{\circ} \mathrm{C}$ comparados as outras estudadas, 50 e 60ㄷ (CARNEIRO et al., 2010).

Todavia, existem estudos que demonstram efeitos positivos da imersão em água a 80 드 no percentual germinativo. Tratamentos de pré-semeadura foram testados na germinação de Albizia richardiana (Voigt) King e Prain e Lagerstroemia speciosa (L.) Pers. e foi observado que a imersão em água a $80^{\circ} \mathrm{C}$ por 10 minutos proporcionou $96 \%$ de germinação para A. richardiana e 64\% para L. speciosa (AZAD et al. 2010). Os mesmos tempos e temperaturas de imersão ocasionaram $82,07 \%$ de germinação de sementes de Albizia procera (Roxb.) Benth. (AZAD et al., 2012).

Métodos de superação de dormência foram avaliados na germinação de Tachigali vulgaris por Silva e Lima, sendo o alto tempo de imersão em água a $80^{\circ} \mathrm{C}$ o provável motivo para o baixo percentual (ABREU et al., 2017). Combinações entre tempos (10, 30 e 60 segundos) e temperaturas ( 80 e 100ㄷ) de imersão em água para sementes de Piptadenia moniliformis Benth foram melhores que o tratamento controle (sementes 
intactas) e não diferiram entre si, mas apresentaram baixos percentuais (FERREIRA et al., 2014).

As imersões das sementes em água sob temperatura ambiente por $24 \mathrm{~h}$, a 40 으 C durante 10 minutos e a $60^{\circ} \mathrm{C}$ por cinco minutos apresentaram índices de velocidade melhores que os demais. Em estudo que avaliou a emergência de plântulas de Astrocaryum aculeatum G. May em resposta à temperaturas e períodos de embebição das sementes, foi observado melhor índice de velocidade para a imersão em água sob temperatura que variou de 25 a 40C (NAZÁRIO et al., 2010).

Testes pré-germinativos foram aplicados em sementes de Samanea tubulosa (Bentham) e não foi observado efeito significativo da imersão em água de 60 a $80^{\circ} \mathrm{C}$, resultando em índices variando entre 1 e 2 (OLIVEIRA et al., 2012). Por outro lado, o aumento da temperatura influenciou de maneira significativa a germinação de sementes de Myrsine parvifolia A. DC em função de temperaturas de 15 a 30 으, sendo encontrados índices de velocidade variando de 0,80 a 1,30 (RIBEIRO et al., 2015).

A imersão das sementes a uma temperatura de $800^{\circ} \mathrm{C}$, em ambos os períodos, reduziu significativamente o índice de velocidade de emergência para $P$. angulata. As imersões de sementes de $S$. tubulosa em água a 60,70 e $80^{\circ} \mathrm{C}$ apresentaram resultados estatisticamente menores que os demais tratamentos, com exceção do tratamento controle (OLIVEIRA et al., 2012).

O menor tempo médio observado no controle pode ser explicado pela não imersão das sementes a altas temperaturas, tornando a emergência mais rápida, o que é interessante do ponto de vista ecológico, pois baixos tempos médios de germinação e emergência permitem que as plantas se estabeleçam de maneira mais rápida e aproveitem condições ambientais mais favoráveis (NOGUEIRA et al., 2010).

A imersão das sementes em água a 80 ㄷ C aumentou o tempo médio de emergência independente do tempo de exposição. Esses resultados podem estar relacionados aos possíveis danos internos na estrutura das sementes (ALVES et al., 2004), o que reduziu o número de sementes emergidas e aumentando o tempo médio de emergência de plântulas.

Resultados contrastantes foram observados no tempo médio de emergência de $A$. aculeatum, que sofreu efeito significativo da interação entre temperaturas de $25,30,35$ e 40 ㄷ C e tempos de imersão de dois, quatro e seis dias, sendo observado menor tempo médios para sementes submetidas a quatro dias de imersão em água a 40C (NAZÁRIO et al., 2010). Para Leucaena leucocephala Lam., as imersões em água a 60 e 80 ㄷ C não prejudicaram o tempo médio de emergência, mas a 100 ㄷ C foi encontrado tempo médio estatisticamente maior (OLIVEIRA et al., 2007).

Por outro lado, não foi constatado efeito significativo do tempo de imersão em água na germinação e no vigor de mudas de copaíba (Copaifera lucens Dwyer), em estudo no qual as sementes foram imersas em água durante 0, 2, 4 e 8 dias e obtiveram tempos médios de germinação de 22,0, 20,3, 25,4 e 24,9 dias, respectivamente, indicando que o tempo de imersão não influencia a germinação de sementes (VIDAL et al., 2014).

A imersão das sementes em água durante $24 \mathrm{~h}$ sob temperatura ambiente proporcionou maior velocidade média de emergência, o que pode ser explicado pelo fato de que essas sementes não foram submetidas ao aumento de temperatura. A alta temperatura afeta de maneira negativa os mecanismos 
fisiológicos das sementes e a viabilidade do embrião, atrasando a germinação da semente e provocando sua morte (OLIVEIRA et al., 2007).

A temperatura de imersão de 80 으 durante 10 minutos ocasionou menor velocidade média de emergência. Temperaturas acima da faixa ótima, a princípio, aceleram a germinação, mas com a interrupção da exposição, diminui o número de sementes que podem germinar (CARVALHO et al., 2012), o que explica a baixa velocidade média, visto que esse tratamento apresentou menor percentual de emergência.

\section{CONCLUSÕES}

A imersão das sementes de Physalis angulata L. em água sob temperatura ambiente durante $24 \mathrm{~h}$ pode ser indicada para o sucesso na emergência de plântulas, pois proporciona maior e mais rápida emergência de plântulas. A imersão em água a 40 ํ C durante dez minutos e a $60{ }^{\circ} \mathrm{C}$ por cinco minutos são alternativas que também apresentam bons resultados. A temperatura de imersão em água a 80 으 prejudica drasticamente a emergência de plântulas, não sendo recomendável para aplicação em práticas prégerminativas para a cultura.

\section{REFERÊNCIAS}

ABREU, D. C. A. D.; PORTO, K. G.; NOGUEIRA, A. C.. Methods of breaking dormancy and germination substrates for Tachigali vulgaris L. G. Silva \& H. C. Lima. Floresta e Ambiente, v. 24, e00071814, 2017.

ALVES, A. U.; DORNELAS, C. S. M.; BRUNO, R. D. L. A.; ANDRADE, L. A. D.; ALVES, E. U.. Superação da dormência em sementes de Bauhinia divaricata L. Acta Botânica Brasílica, v.18, n.4, p.871-879, 2004.

AZAD, M. S.; BISWAS, R. K.; MATIN, M. A.. Seed germination of Albizia procera (Roxb.) Benth. in Bangladesh, a basis for seed source variation and pre-sowing treatment effect. Forestry studies in China, v.14, n.2, p.124-130, 2012.

AZAD, S.; PAUL, N. K.; MATIN, A.. Do pre-sowing treatments affect seed germination in Albizia richardiana and Lagerstroemia speciosa?. Frontiers of Agriculture in China, v.4, n.2, p.181-184, 2010.

BASKIN, C. C.; BASKIN, J. M.. Seeds, ecology, biogeography, and evolution of dormancy and germination. 2 ed. San Diego: Academic Press, 2014.

BENEDITO, C. P.; RIBEIRO, M. C. C.; TORRES, S. B.; GUIMARÃES, I. P.; OLIVEIRA, K. J. B.. Dormancy overcoming, temperatures and substrates on germination of Mimosa tenuiflora Willd seeds. Semina: Ciências Agrárias, v.38, p.125-134, 2017.

BRASIL. Ministério da Agricultura, Pecuária e Abastecimento. Regras para análise de sementes. Brasília: Mapa, 2009.

CARNEIRO, G. G.; BARBOSA, J. A.; SILVA, E. O.; GOIS, G. C.; LUCENA, H. H.; ALVES, E. U. Germinação de pimentas Cambuci submetidas à superação de dormência em água quente. Bioscience Journal, v.26, n.6, p.882-885, 2010.
CARVALHO, N. M.; NAKAGAWA, J.. Sementes: ciência, tecnologia e produção. 5 ed. Jaboticabal: FUNEP, 2012.

CASTRO, D. S.; ARAUJO, E. F.; BORGES, E. E. L.; AMARO, H. T. R.. Caracterização da testa de sementes de Apuleia leiocarpa ([Vogel] J.R. Macbr) após superação de dormência. Ciência Florestal, v.27, n.3, p.1061-1068, 2017.

EDMOND, J. B.; DRAPALA, W. J.. The effects of temperature, sand, soil, and acetone on germination of okra seeds. Proceedings of the American Society for Horticultural Science, v.71, n.5, p.428-434, 1958.

FERREIRA, D. F.. Sisvar, a computer statistical analysis system. Ciência e Agrotecnologia, v.35, n.6, p.1039-1042, 2011.

FERREIRA, E. G. D. S.; MATOS, V. P.; SILVA, R. B.; SANTOS, H. H.; SENA, L. H.. Thermal scarification to overcome Piptadenia moniliformis seeds dormancy. Revista Brasileira de Ciências Agrárias, v.9, p.79-83, 2014.

KOGER, C. H.; REDDY, K. N.; POSTON, D. H.. Factors affecting seed germination, seedling emergence, and survival of texasweed (Caperonia palustris). Weed Science, v.52, n.6, p.989-995, 2004.

KOTOWISKI, F.. Temperature relations to germination of vegetable seeds. Proceedings of the American Society of Horticultural Science, v.23, p.176-184, 1926.

KUSUMANINGTYAS, R. W.; LAILY, N.; LIMANDHA, P.. Potential of ciplukan (Physalis angulata L.) as source of functional ingredient. Procedia Chemistry, v.14, p.367-372, 2015.

MAGUIRE, J. D.. Speed of germination aid in selection and evaluation for seedling emergence and vigor. Crop Science, 


\section{v.2, n.2, p.176-77, 1962}

MOTSA, M. M; SLABBERT, M. M.; AVERBEKE, W. V.; MOREY, L.. Effect of light and temperature on seed germination of selected African leafy vegetables. South African Journal of Botany, v.99, p.29-35, 2015.

NAZÁRIO, P.; FERREIRA, S. A. N.. Emergência de plântulas de Astrocaryum aculeatum $\mathrm{G}$. May. em função da temperatura e do período de embebição das sementes. Acta amazônica, v.40, p.165-170, 2010 .

NOGUEIRA, F. C. B.; MEDEIROS FILHO, F.; GALLÃO, M. I.. Caracterização da germinação e morfologia de frutos, sementes e plântulas de Dalbergia cearensis Ducke (pauvioleta)-Fabaceae. Acta Botânica Brasílica, v.24, n.4, p.978985, 2010

OLIVEIRA, A. B. D.; MEDEIROS FILHO, S.. Influência de tratamentos pré-germinativos, temperatura e luminosidade na germinação de sementes de leucena, cv.

Cunningham. Revista Brasileira de Ciências Agrárias, v.2, n.4, p.268-274, 2007

OLIVEIRA, L. M.; BRUNO, R. L. A.; ALVES, E. U.; SOUSA, D. M.
M.; ANDRADE, A. P.. Tratamentos pré-germinativos em sementes de Samanea tubulosa Bentham - (LeguminoseaeMimosoideae). Revista Árvore, v.36, n.3, p.433-440, 2012.

PATEL, P. R.; GOL, N. B.; RAO, T. V. R.. Physiochemical changes in sunberry (Physalis minima $\mathrm{L}$.) fruit during growth and ripening. Fruits, v.66, p.37-46, 2011

RIBEIRO, J. N. S.; COSTA, C. S. B.. The effect of temperature regulation on seed germination of the tropical tree Myrsine parvifolia A. DC near its southern limit. South African Journal of Botany, v.98, p.128-133, 2015.

SOUZA, M. O.; SOUZA, C. L. M.; PELACANI, C. R.; SOARES, M.; MAZZEI, J. L.; RIBEIRO, I. M.; RODRIGUES, C. R.; TOMASSINI, T. C. B.. Osmotic priming effects on emergence of Physalis angulata and the influence of abiotic stresses on physalin content. South African journal of botany, v.88, p.191-197, 2013.

VIDAL, D. B.; ANDRADE, I. L; ANDRADE, E. L. P.; MIELKE, M. S. Effects of submergence in water on seed germination and vigor of the Copaifera lucens (Fabaceae) seedlings. Journal of Forestry Research, v.25, n.4, p.903-908, 2014. 